\title{
The Strengths And Difficulties Questionnaire (SDQ) as a dimensional measure of child mental health
}

\author{
Anna Goodman, ${ }^{1}$ Robert Goodman ${ }^{2}$ \\ 1 London School of Hygiene \& Tropical Medicine, London \\ 2 King's College London Institute of Psychiatry, London.
}

Corresponding author: Anna Goodman, Department of Epidemiology and Population Health, London School of Hygiene and Tropical Medicine, Keppel Street, London, WC1e 7HT, UK. Email address: anna.goodman@lshtm.ac.uk

Note: this is a personal version, created by Anna Goodman, of the text of the accepted journal article. It reflects all changes made in the peer review process, but does not incorporate any minor modifications made at the proof stage. The complete citation for the final journal article is:

- Goodman, A; Goodman, R; (2009) Strengths and Difficulties Questionnaire as a Dimensional Measure of Child Mental Health. J Am Acad Child Adolesc Psychiatry, 48 (4). pp. 400-3

- DOI: $10.1097 / C H I .0 b 013 e 3181985068$

Copyright ( $\subset$ and Moral Rights for this paper are retained by the individual authors and/or other copyright owners 


\section{$\underline{\text { Abstract }}$}

Objective: To provide the first explicit evaluation of the dimensionality of the total difficulties score of the Strengths and Difficulties Questionnaire (SDQ), a widely used measure of child mental health. We do so by validating the SDQ across its full range against the prevalence of clinical disorder.

Method: We use two large $(\mathrm{N}=18,415)$, nationally-representative surveys of children and adolescents aged 5-16 in the general British population. SDQs were completed separately by parents, teachers and children aged 11-16, and children also received a multi-informant clinician-rated clinical diagnosis. 7,912 children from the baseline survey were also re-assessed for clinical diagnosis at three-year follow-up.

Results: Across the full range of the parent, teacher and child SDQ, children with higher total difficulty scores have greater psychopathology as judged by the prevalence of clinical disorder. This was true cross-sectionally and also in predicting to disorder-status three years later. There was no evidence of threshold effects for the SDQ at either high or low scores, but rather the odds of disorder increased at a constant rate across the range (odds ratios between 1.14 and 1.28 per one-point increase in SDQ score).

Conclusion: Our findings support the use of the SDQ as a genuinely dimensional measure of child mental health.

Keywords: Strengths and Difficulties Questionnaire; child and adolescent; psychopathology; questionnaire; psychometric 


\section{Introduction}

The Strengths and Difficulties Questionnaire (SDQ) is a brief screening questionnaire for child mental health problems 12 which can be completed by parents, teachers and children. It is widely used for research purposes in the US and other high, middle and low-income countries and there has also been recent interest in using it to monitor the effectiveness of routine clinical services or as a measure of child well-being in community settings such as schools.

Yet like most measures of psychopathology, the SDQ has thus far primarily been validated in terms of its ability to distinguish between clinic and community samples 1 or as a screening device to detect children with a mental health disorder.2 3 That the SDQ performs well at these tasks is evidence that children with 'high' SDQ scores have greater rates of mental disorder than children with 'low' SDQ scores, and is sufficient to justify its utility as a screening device for identifying high-risk children. Yet it does not imply that differences in mean SDQ scores between groups, or a greater change in mean SDQ score in an intervention than a control group, necessarily correspond to differences in mental health. For example, there could be threshold effects such that children with a score of below (say) 10 all had undifferentiatedly good mental health. If so, then an intervention could move a group of children from having a score of 8 to having a score of 4 without any genuine improvement in child well-being. Thus only if one knows that a lower SDQ score anywhere across the SDQ's range corresponds to lower psychopathology can one legitimately infer that lower mean SDQ scores in one group compared to another really do reflect a mental health advantage or an effective mental health intervention. Moreover, in order to model the SDQ and interpret changes in mean score correctly, it is also useful to know whether the magnitude of this change is constant across the range of the SDQ..

We therefore sought to evaluate whether the parent, teacher and child SDQ are dimensional measures of child mental health across their full range, judging child psychopathology using a multi-informant clinical diagnosis. To our knowledge, this is the first evaluation of its kind for the SDQ. We present analyses using clinical diagnoses assigned at the same time as the SDQ scores and also analyses using clinical diagnoses assigned three years later. Our rationale for including the latter was to exclude the possibility that an association between SDQ score and contemporaneous clinical diagnosis could be due to transient rater biases.

\section{Method}

\section{Measures}

The Strengths and Difficulties Questionnaire (SDQ) is a measure of mental health problems in children aged 4-17 which can be administered to parents, teachers and to children aged 11 or over. 12 The 20 items relating to emotional symptoms, conduct problems, hyperactivity and peer problems are summed to create a 'total difficulty score' ranging from 0-40. The Development and Well-Being Assessment (DAWBA) is a 
detailed psychiatric interview for parents and children aged 11-16, and a briefer questionnaire for teachers. 5 It includes a fully-structured section (including screening questions and skip rules) followed by open-ended descriptions by respondents of any problem areas. Experienced clinicians then use both the closed and open DAWBA responses, and triangulate information from across informants, in order to assign diagnoses according to DSM-IV.6

\section{Sample}

Our data comes from the British Child and Adolescent Mental Health Surveys (BCAMHS) of 1999 and 2004. These were two nationally-representative surveys of the mental health of children and adolescents aged 5-16 in Great Britain, which have previously been described in detail.4 7 Both surveys were population-based, using the British Child Benefit Register as a sampling frame; in Britain child benefit is a universal state benefit payable for each child in the family with extremely high uptake. Parents were approached to participate and, with parental permission, the child's teacher and children aged 11-16 were also approached. All participants were administered the appropriate DAWBA and SDQ by trained lay interviewers, and a small team of experienced child psychiatrists subsequently assigned clinical diagnoses using the DAWBA interviews from all available participants. The clinicians had access to SDQ scores when making baseline diagnoses, but diagnostic decisions were made based on the far more detailed questions in the DAWBA. The clinicians were blind to baseline SDQ scores when making diagnoses at 3-year follow-up.

Between the two B-CAMHS surveys 26,544 children were selected and their principal caregivers ('parents') approached, of whom 18,415 (69\%) participated (10 438 in BCAMHS99, 7,977 in B-CAMHS04). These children had a mean age of 10.2 years and were $50.8 \%$ male. All 18,415 children were assigned a DAWBA diagnosis (including 'no disorder'), and SDQ total difficulty scores were available from 18224 parents, 14,313 teachers and 7,678 youths aged 11-16.

Both B-CAMHS surveys also included a three-year follow-up. B-CAMHS99 attempted to follow up all children who had a disorder at baseline (response rate $582 / 983$ or $59.2 \%$ ) and a random third of the children without a disorder (response rate 2,004/3,081 or 65.0\%).8 B-CAMHS04 attempted to follow up all children in the baseline survey (response rate 5,326/7,977=66.8\%).9 Of the combined sample of 7,912 children who were assigned a DAWBA diagnosis at three year follow-up, baseline SDQ data was available from 7,901 parents, 6,266 teachers and 3,408 youths.

Ethical approval was granted by the Institute of Psychiatry research ethics committee, London, for B-CAMHS99, and by the British multicentre research ethics committee for B-CAMHS04. 


\section{Data analysis}

For children with any given SDQ score we calculated the prevalence of clinical disorder (defined as receiving at least one DAWBA diagnosis) at baseline and follow-up. We repeated these analyses for the parent, teacher and youth SDQs. Prevalence estimates at the very high end of the distribution were unstable because of small numbers of children with each SDQ score. For the purposes of graphical presentation, we therefore grouped all high scores once the number of children per SDQ point fell to ten or fewer. Follow-up analyses use probability weighting to adjust for the fact that only a third for children without a disorder at baseline in B-CAMHS99 were selected to be approached for followup, compared with $100 \%$ of children in B-CAMHS04 or with a disorder in B-CAMHS99. All analyses were conducted using STATA.10.

We fitted logistic regression models with DAWBA diagnosis as the outcome and with the parent, teacher or youth SDQ score as an explanatory variable. In order to test whether an increase in SDQ corresponded with a constant increase in the odds ratio of disorder across the SDQ's range, we used likelihood ratio tests to compare models in which SDQ score was entered as a linear variable with models in which it was entered as a categorical variable (in one-point bands).

\section{$\underline{\text { Results }}$}

Figure 1 presents the prevalence of DAWBA diagnosis for parents, teachers and children at baseline and follow-up. The general pattern for all three informants is for each onepoint increase in SDQ score to correspond to an increase in the prevalence of DAWBA diagnosis. This is true both of DAWBA diagnosis measured contemporaneously at baseline and three years later. For no informant is there any suggestion of a threshold effect for either very low or very high scores. 
Figure 1: Prevalence of child mental disorder and $95 \%$ confidence intervals at baseline and threeyear follow-up by parent, teacher and child SDQ scores.
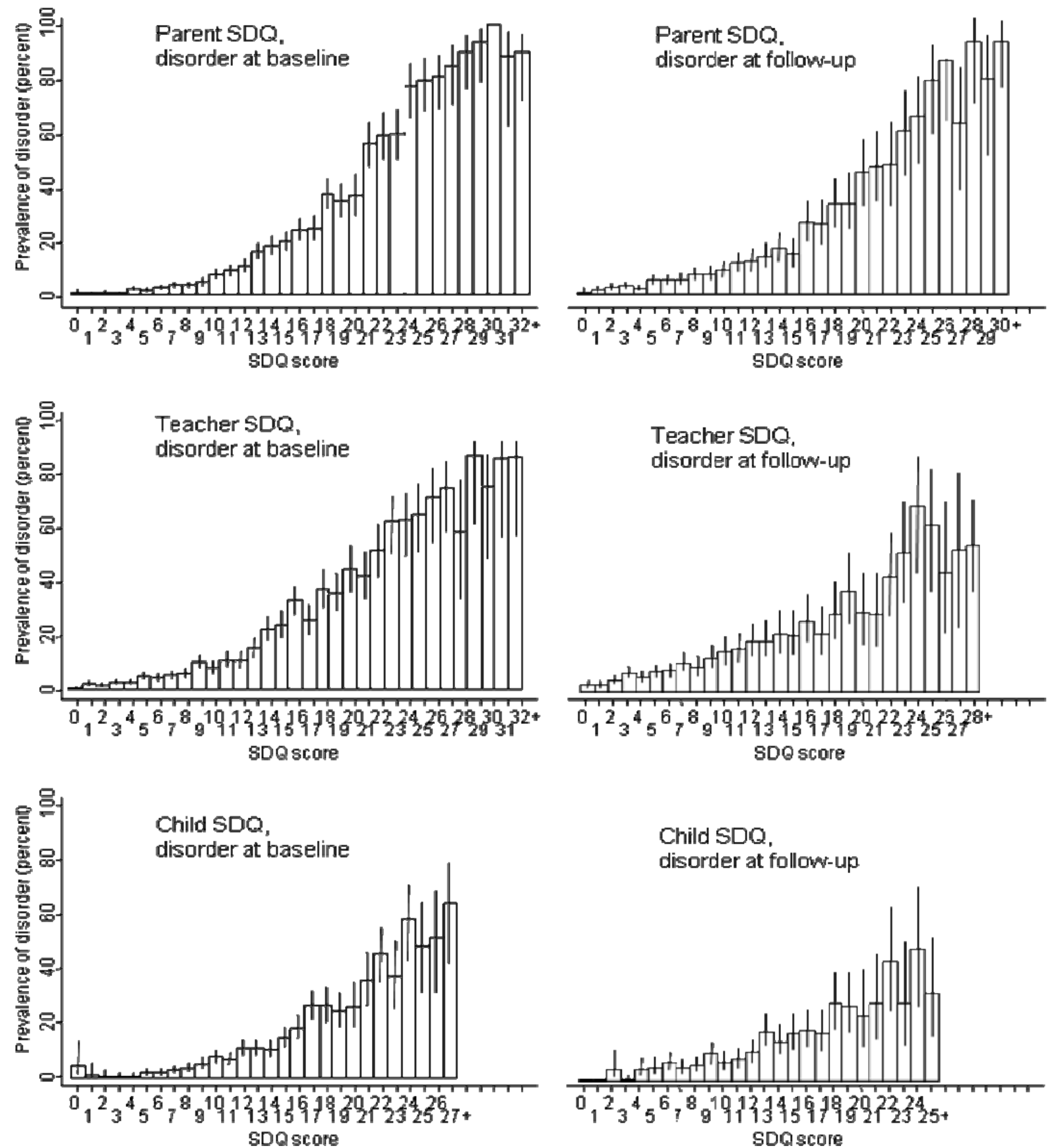

We grouped all high scores once the number of children per SDQ point fell to ten or fewer, in order to prevent very small numbers leading to instability in the prevalence estimates. The last prevalence estimate presented for each informant and at each time point therefore corresponds to the prevalence for that number of SDQ points or above.

In no instance was there evidence that the model in which the SDQ was entered as a categorical variable provided a better fit than the model in which it was entered as a 
linear term ( $p>0.1$ in all six cases). In Table 1 we therefore report the estimates from the linear model as to the odds ratio for disorder per one-point increase in SDQ. The odds ratios are greater for parents than teachers or youths, and greater at baseline than followup. Note that, as is visible in the exponential shape of the graphs in Figure 1, a constant increase in an individual's relative risk of disorder per one-point increase translates into a greater absolute increase in disorder prevalence at the high end of the range. .

Table 1: Odds ratio for disorder per one-point increase in the SDQ

\begin{tabular}{|l|l|l|}
\hline & $\begin{array}{l}\text { OR for disorder at } \\
\text { baseline }(95 \% \mathrm{CI})\end{array}$ & $\begin{array}{l}\text { OR for disorder at } \\
\text { follow-up }(95 \% \mathrm{CI})\end{array}$ \\
\hline Parent & $1.28(1.27-1.29)$ & $1.21(1.19-1.22)$ \\
\hline Teacher & $1.23(1.22-1.24)$ & $1.14(1.13-1.16)$ \\
\hline Child & $1.23(1.21-1.25)$ & $1.16(1.13-1.18)$ \\
\hline
\end{tabular}

\section{$\underline{\text { Discussion }}$}

Our findings indicate that across the full range of the parent, teacher and youth SDQ, children with higher total difficulty scores have greater psychopathology as judged by the prevalence of clinical disorder. This is true cross-sectionally and also in predicting to disorder-status three years later. There was no evidence of threshold effects for the SDQ at either high or low scores, but rather the odds of disorder increased at a constant rate across the range (odds ratios between 1.14 and 1.28 per one-point increase in SDQ score).

Our results therefore support the use of the SDQ as a dimensional measure of child mental health for comparing average scores in child mental health between groups, over time or following an intervention. The dimensionality of the SDQ also means that it could be amenable to other uses such as not simply splitting children into 'high' and 'low' risk groups, but instead taking a more graded approach which differentiates between different risk groups. One interesting question for future studies is whether the SDQ score also behaves dimensionally as judged against measures of the presence of positive psychological well-being.

The results we present in this paper relate to the prevalence of disorder at baseline and at three-year follow-up in children whose SDQ scores were measured only once. These analyses therefore do not address the question of how to interpret changes in SDQ scores for a particular individual over time, or following an intervention. An individual child attending a mental healthclinic may show a decrease in SDQ score upon repeated measurement even in the absence of an improvement in their psychopathology because of factors such as regression to the mean or a tendency for informants to report more problems in the first interview than subsequently. We discuss these considerations in detail in Ford et al.10 and provide a preliminary evaluation of an 'Added Value' score which removes these artefactual sources of change in a child's SDQ score over time.

Our results therefore do not imply that any change in an individual or group's SDQ score necessarily represent a change in their mental health. Our results do, however, indicate that differences in mean scores between groups (including intervention and control 
groups) can legitimately be interpreted as reflecting genuine differences in mental health. Of course, this dimensionality still does not indicate whether the benefit applies to all children, or only to high- or low-risk children. It does, however, imply that the mental health of some children has improved. This assumption is implicit in many research or evaluation analyses, and it is therefore important and reassuring to conclude that it does seem to be justified.

\section{Conflict of interest}

AG and RG are directors and part owners of Youthinmind, which provides no-cost and low-cost software and web sites related to the SDQ and the DAWBA.

\section{Acknowledgements}

The British Child and Adolescent Mental Health Surveys of 1999 and 2004 and their longitudinal extensions were funded by the Department of Health and the Scottish Executive, and carried out by the Office for National Statistics. AG is supported by an ESRC/MRC research degree studentship.

\section{References}

1. Goodman R. The Strengths and Difficulties Questionnaire: a research note. J Child Psychol Psychiatry 1997;38(5):581-6.

2. Goodman R. Psychometric properties of the strengths and difficulties questionnaire. J Am Acad Child Adolesc Psychiatry 2001;40(11):1337-45.

3. Goodman R, Renfrew D, Mullick M. Predicting type of psychiatric disorder from Strengths and Difficulties Questionnaire (SDQ) scores in child mental health clinics in London and Dhaka. Eur Child Adolesc Psychiatry 2000;9(2):129-34.

4. Ford T, Goodman R, Meltzer H. The British Child and Adolescent Mental Health Survey 1999: the prevalence of DSM-IV disorders. J Am Acad Child Adolesc Psychiatry 2003;42(10):1203-11.

5. Goodman R, Ford T, Richards H, Gatward R, Meltzer H. The Development and WellBeing Assessment: description and initial validation of an integrated assessment of child and adolescent psychopathology. J Child Psychol Psychiatry 2000;41(5):645-55.

6. American Psychiatric Association. Diagnostic and Statistical Manual of Mental Disorders, 4th edition (DSM-IV). Washington, DC: American Psychiatric Association., 1994.

7. Green H, McGinnity A, Meltzer H, Ford T, Goodman R. Mental health of children and young people in Great Britain, 2004: Palgrave MacMillan, 2005.

8. Meltzer H, Gatward R, Corbin T, Goodman R, Ford T. Persistence, onset, risk factors and outcomes of childhood mental disorders. London: The Stationery Office, 2003.

9. Parry-Langdon et al. ONS report to be published in September 2008, In preparation. 10. Ford T, Hutchings J, Bywater T, Goodman A, Goodman R. The Strengths and Difficulties Questionnaire Added Value Score. Br J Psychiatry In press. 